\title{
EL PRIMER MOVIMIENTO MIGRATORIO EN LA BIOGRAFÍA DE LOS ESPAÑOLES
}

\author{
POR \\ M. ${ }^{a}$ DOLORES PUGA GONZÁLEZ y ANTONIO ABELLÁN GARCÍA
}

\section{Introducción}

Los movimientos migratorios han tenido una gran importancia en la actual composición y distribución de la población española. Aunque han existido desde antiguo y las migraciones a ultramar arrastraron importantes contingentes de población tanto a finales del XIX como a principios del xx, la movilidad interna de grandes flujos de población cobra verdadera importancia a lo largo del siglo $\mathrm{xx} \mathrm{y}$, en concreto, a partir de la segunda década del mismo.

Numerosos autores han prestado atención a este fenómeno por sus destacadas consecuencias demográficas, con efecto tanto sobre la estructura como sobre la dinámica de las poblaciones de origen y destino, así como por su relación con el intenso proceso de urbanización y despoblamiento rural que se ha producido. Ha sido estudiado de forma indirecta a partir de saldos migratorios (a nivel provincial, de partido judicial, e incluso municipal), debido a las dificultades de obtención de otros datos más precisos (García Barbancho, Puyol, etc.). Se conocen las cifras, la importancia del fenómeno, los efectivos aproximados desplazados en cada década y los lugares de origen y destino. Pero quedan

M. ${ }^{a}$ Dolores Puga González y Antonio Abellán García: Instituto de Economía y Geografía. Consejo Superior de Investigaciones Científicas.

Estudios Geográficos

Tomo LIX, n. ${ }^{\circ} 233$, octubre-diciembre 
algunas cuestiones por resolver: no se conocen las características en el momento de la migración, algunas fundamentales como la edad o si se realizó en compañía de toda o parte de la familia, ni las motivaciones declaradas, la relación con otros eventos biográficos, o el número de desplazamientos, como tampoco si existe un patrón similar de movilidad entre las diferentes generaciones.

Este artículo pretende un análisis del fenómeno migratorio desde la óptica del curso de vida de sus protagonistas: es un estudio longitudinal de la historia migratoria de las tres primeras cohortes del siglo (los nacidos en 1901-10, 1911-20 y 1921-30), es decir, aquellos individuos que tenían en 1991 (fecha de la fuente del estudio) 60 o más años y, por tanto, una ya extensa historia personal, muchos de ellos habían cerrado su fase laboral o estaban a punto de concluirla, y fueron en parte los protagonistas de una de las mayores oleadas migratorias de la historia de España.

Se estudia su trayectoria migratoria, y más en concreto su primer movimiento, que para la mayoría de ellos fue el único y para casi todos el más importante y de mayores consecuencias biográficas. Se trata, pues, de situar la movilidad de estos individuos no sólo en el momento cronológico o histórico en que se produce, sino también en el momento biográfico: edad del sujeto en el momento del cambio de residencia, rango de la migración, relación con otros eventos del curso de vida. Es un intento de acercarse al comportamiento migratorio, de considerar la migración en la trayectoria vital de sus protagonistas. En estudios posteriores se abordará la historia migratoria completa, la dirección de los flujos, el tipo de hogar o familia migrante y las razones declaradas para esa movilidad.

\section{Fuente y metodología}

La fuente de datos del estudio es la Encuesta Sociodemográfica (ESD) realizada por el Instituto Nacional de Estadística en el último cuatrimestre de 1991, y en la que se entrevistaron a un total de 159.154 individuos mayores de 10 años residentes en España, de los que 45.566 habían nacido antes de 1931. Esta muestra permite un nivel de desagregación temporal (probabilidades anuales) imposible de realizar con las muestras con las que habitualmente se trabaja. La información

$$
-690-
$$


retrospectiva faculta el estudio longitudinal y facilita el conocimiento de algunos aspectos de la historia demográfica reciente hasta ahora difícilmente abarcables. Situar los eventos demográficos en la biografía individual abre posibilidades de análisis que son las que se pretenden explorar en este artículo.

Problemas de la fuente.-El primero es, sin lugar a dudas, la enorme complejidad de la misma, lo que ha motivado los escasos estudios basados en ella ${ }^{1}$. El segundo es que, al tratarse de una encuesta retrospectiva, la muestra no es representativa, en realidad, de la cohorte inicial sino de los supervivientes de esa cohorte residentes en España en 1991. Esto supone dos problemas derivados:

a) la emigración al extranjero tuvo efectos diferenciales tanto a nivel temporal como geográfico. La emigración hacia América, de gran importancia a principios de siglo, se redujo progresivamente en favor de la emigración hacia Europa (con mayor tasa de retorno que los movimientos hacia América) y fundamentalmente, de las migraciones interiores. Se reconoce un efecto de subestimación de efectivos iniciales debido a la existencia de emigración exterior no retornada (y que está fuera de la muestra entrevistada), mayor en la primera de las cohortes que en las más jóvenes.

b) la interferencia de la mortalidad en las tablas de migrabilidad, al ser la población inicial de la tabla una reconstrucción de la misma a partir de los supervivientes. En este caso, se ha asumido, para la construcción de las tablas, que las tasas de mortalidad no han tenido diferente incidencia entre migrantes y sedentarios, es decir, en el numerador (migrantes) y en el denominador (migrantes más sedentarios).

La metodología utilizada ha sido la construcción de tablas de vida, en este caso, tablas de migrabilidad, para cada una de las cohortes y orden de rango del movimiento, presentándose aquí los resultados para la primo-movilidad o primer movimiento migratorio de todos aquellos que migraron alguna vez. Como variable temporal se ha utilizado la duración de la sedentariedad, que para el primer movimiento coincide,

${ }^{1}$ Queremos hacer constar nuestro agradecimiento al Centre d'Estudis Demogràfics (Barcelona), y en concreto a Marc Ajenjo, por la desinteresada cesión del complejo programa de control para la lectura de las variables de la encuesta, por el paquete estadístico SPSS. 
obviamente, con la edad en el momento de la migración. A partir de ellas se han obtenido las probabilidades de movilidad por edades simples, así como las acumuladas, para las tres cohortes. Se considera la movilidad como cambio de municipio de residencia, es decir, con traspaso de fronteras administrativas. A efectos de este trabajo, ese evento biográfico será denominado indistintamente movilidad o migración.

\section{Movilidad biográfica e intensidad migratoria}

Se considera como movilidad biográfica el número de movimientos realizados a lo largo de la vida por un individuo. Los españoles nacidos en las tres primeras décadas del siglo han realizado muy pocos movimientos en su vida. Frente a la alta movilidad biográfica de la población de países anglosajones, las sociedades latinas muestran una tradicional sedentariedad. La gente apenas se mueve, y los que lo hacen, se mueven pocas veces. Las generaciones aquí estudiadas no son, como era de esperar, ninguna excepción a este comportamiento. Prácticamente la mitad de los nacidos en esas décadas no se movió jamás del municipio de nacimiento $(50.8 \%, 47.5 \%$ y $46.7 \%$ para las tres cohortes consideradas, respectivamente), por lo que la media de movimientos por individuo, incluyendo a los sedentarios, se sitúa por debajo de la unidad en la primera cohorte y muy próxima en las otras (Cuadro I).

De todos los que han realizado algún movimiento en su vida, más de la mitad efectuaron tan sólo uno, mientras que los que migraron tres o más veces suponen en torno al $10 \%$ de las cohortes. Es decir, la migración ha sido un evento biográfico poco frecuente para estos individuos; pocos son los que han hecho las maletas dos veces y muy raros los que han decidido -o se han visto obligados- a cambiar de municipio de residencia con mayor frecuencia. Tomando la media por individuo, calculada tan sólo para las personas que realizaron algún movimiento, de modo que no se vea afectada por la intensidad migratoria de la cohorte, se sitúa en 1.8, 1.9 y 1.9 desplazamientos, respectivamente.

Existe un bajo nivel de movilidad biográfica y una gran estabilidad temporal o histórica de este comportamiento, que prácticamente no varía en los individuos considerados. La mencionada tendencia a la sedentariedad constituye un comportamiento arraigado en la tradición cultural y en las formas de vida, por lo que cambia muy lentamente. 
Cuadro I

MOVILIDAD BIOGRÁFICA POR COHORTES DE NACIMIENTO. ESPAÑA (1901-1930)

\begin{tabular}{|c|c|c|c|}
\hline $\begin{array}{c}N^{\circ} \\
\text { movimientos }\end{array}$ & $\begin{array}{c}1901-10 \\
\%\end{array}$ & $\begin{array}{c}\text { COHORTE } \\
1911-20 \\
\%\end{array}$ & $\begin{array}{c}1921-30 \\
\%\end{array}$ \\
\hline 0 & 50.8 & 47.5 & 46.7 \\
\hline 1 & 27.2 & 28.3 & 28.6 \\
\hline 2 & 12.8 & 13.9 & 14.2 \\
\hline 3 & 4.7 & 4.9 & 5.3 \\
\hline 4 & 2.0 & 2.6 & 2.4 \\
\hline 5 & 1.0 & 1.3 & 1.2 \\
\hline 6 & 0.6 & 0.6 & 0.7 \\
\hline 7 & 0.4 & 0.5 & 0.4 \\
\hline 8 & 0.2 & 0.2 & 0.3 \\
\hline 9 & 0.1 & 0.1 & 0.1 \\
\hline 10 & 0.1 & 0.1 & 0.1 \\
\hline 11 & 0.1 & 0.1 & 0.1 \\
\hline 12 & 0.0 & 0.0 & 0.0 \\
\hline Media & 0.9 & 1.0 & 1.0 \\
\hline $\begin{array}{l}\text { Media } .^{\circ} \text { mov } \\
\text { (sólo migrantes) }\end{array}$ & 1.8 & 1.9 & 1.9 \\
\hline
\end{tabular}

FUENTE: Elaboración propia a partir de los datos primarios de la ESD.

En cambio, la intensidad migratoria muestra una tendencia creciente para las cohortes. Es decir, en cada una de ellas el número de individuos que decide realizar, al menos, un desplazamiento es creciente con respecto a la anterior. La tasa bruta de migración calculada para el primer movimiento, que es de un 5.5 por mil anual para la cohorte nacida durante la primera década del siglo, alcanza ya un 7.6 para los nacidos 20 años más tarde (cuadro II). Los individuos nacidos en los años veinte no decidieron realizar un mayor número de movimientos que los nacidos décadas atrás, sin embargo, cambiaron alguna vez de municipio un número creciente de ellos, constituyendo generaciones «más migratorias» que las anteriores.

Conviene señalar que la emigración hacia América afectó más a la primera de las cohortes; aunque su máximo se produjo entre 1905 y 1914 
CUADRO II

TASA BRUTA ANUAL DE MIGRACIÓN

\begin{tabular}{c|c}
\hline COHORTE & $m$ (por mil) \\
\hline $1901-10$ & 5.5 \\
$1911-20$ & 6.6 \\
$1921-30$ & 7.6 \\
\hline
\end{tabular}

FUENTE: Elaboración propia a partir de los datos primarios de la ESD.

(más de 1.650.000), siguió siendo significativa hasta los años veinte (Nicolau, 1989; García Fernández, 1965), y perdió gran capacidad de atracción para las dos siguientes cohortes. Este flujo a ultramar se reanudó a finales de los años cuarenta (siendo especialmente intenso en algunas áreas geográficas, como Galicia, de donde procedían el 50\% de los emigrantes que partieron hacia el otro lado del océano), pero nunca llegó a alcanzar los volúmenes migratorios de principios de siglo; por ejemplo, entre 1947 y 1962 se registraron 44.000 emigrantes anuales, además de contar, en este período, con un mayor porcentaje de retorno. Esta menor intensidad migratoria de la primera cohorte, podría ser consecuencia de la subestimación provocada por esta emigración exterior no retornada y, por tanto, no contabilizada en la Encuesta Sociodemográfica de 1991.

Las probabilidades de migración para cada una de las tres cohortes muestran también un significativo aumento de la intensidad migratoria (Figura 1), alcanzando la curva perteneciente a la tercera probabilidades anuales cercanas al 25 por mil, muy lejos de los máximos en torno a 15 presentados por la primera de las cohortes. En este proceso juegan dos factores, no sólo la intensidad migratoria aumenta de forma global, sino que además se concentra significativamente a lo largo de una veintena de años (1945-65) que, por lo tanto, muestran unas probabilidades mucho más elevadas, tanto por el aumento de la intensidad migratoria como de la concentración temporal.

Posiblemente, los acontecimientos históricos que conoció la generación más joven posibilitaron que sus probabilidades de migración se vieran menos restringidas que las de las anteriores, e incluso potenciadas. De esta situación resulta no sólo una mayor intensidad migratoria, sino también, como se verá, una menor dispersión del primer movi- 


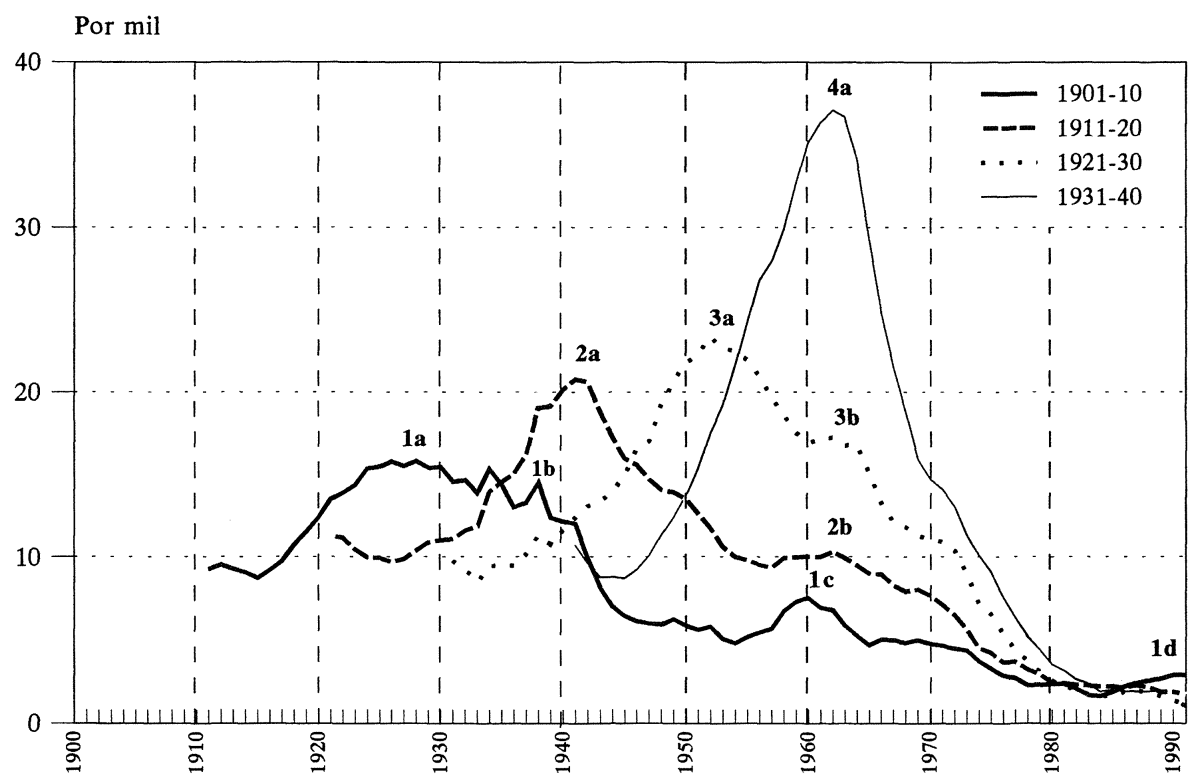

Fuente: Elaboración propia a partir de los datos primarios de la ESD. Nota: Curva suavizada con una media móvil de cinco años.

FIGURA 1.-Primer movimiento migratorio según cohorte de nacimiento. España, 1901-1940 (anual)

miento a lo largo de la vida, concentrado en torno a las clásicas edades de migración.

\section{Evolución histórica}

Los españoles nacidos entre 1901 y 1910 presentan una curva migratoria con mayor dispersión temporal de su primer movimiento, que aunque se centra en los años veinte, sigue siendo significativa hasta 1970 (Figura 1). Los nacidos entre 1911 y 1920 ven su historia migratoria muy determinada por los efectos de la Guerra Civil, con un máximo de movilidad en torno a 1940. La tercera de las cohortes presenta una movilidad más concentrada que las anteriores, entre 1949 y 1960, pero manteniendo unas probabilidades todavía altas hasta 1970. Las tres 
generaciones ven reactivadas sus probabilidades de migración a principios de la década de los sesenta a pesar de las distintas edades en las que se encuentran los individuos de cada una de ellas.

La primera cohorte tiene una movilidad bastante temprana en la segunda década del siglo (en parte ligada a la de sus padres), propiciada por la pequeña «revolución económica» que la guerra europea genera en España. Las condiciones permanentes de crisis en el campo español motivan el trasvase de un gran contingente de población rural (jornaleros), las posibilidades de emigración a América se alejan, a mediados de la década, y por el contrario surge una creciente demanda de brazos en las regiones industriales, debido al aumento de las exportaciones a Europa. Los núcleos industriales comienzan a constituir un foco de atracción, y paralelamente comienza la progresiva aceleración del proceso de urbanización interior. Los niveles de movilidad generales permanecen crecientes durante el primer quinquenio de los años veinte. En este período se produce una fuerte oleada de movilidad interior, protagonizada, por tanto, por los nacidos a finales del xIX y por la primera cohorte de este siglo (Figura 1, marcado como 1a).

En los últimos años veinte y los primeros de la década de los treinta, las áreas industriales, catalana y vasca, se encuentran en su momento de vitalidad más bajo. A esta situación hay que unir el impacto de la gran depresión, que aunque mucho menos profunda en España que en otros países industrializados, hizo notar sus efectos en los principales núcleos industriales. En palabras de Nadal (1984): «nunca hasta entonces se había producido una inflexión semejante en tan corto periodo de tiempo; nunca, sobre todo, la población industrial se había visto tan afectada por una crisis económica: en la ciudad de Barcelona, por ejemplo, ...las dificultades económicas redujeron la venida de inmigrantes». Las probabilidades de movilidad de la primera cohorte, ya estabilizadas desde mediados de los veinte, se reducen.

La Guerra Civil tuvo un impacto en los flujos migratorios: «...cambió el curso de las cosas, provocando una emigración muy nutrida (...) y unos desplazamientos anormales» (Nadal, 1984). De hecho los desplazamientos pueden parecer anormales a partir de las cifras agrupadas por períodos quinquenales o decenales, dado lo inesperado de un comportamiento altamente migratorio en un período de contienda civil; pero resulta mucho más comprensible a partir de las probabilidades anuales, no suavizadas, como se refleja en la figura 1 bis. 


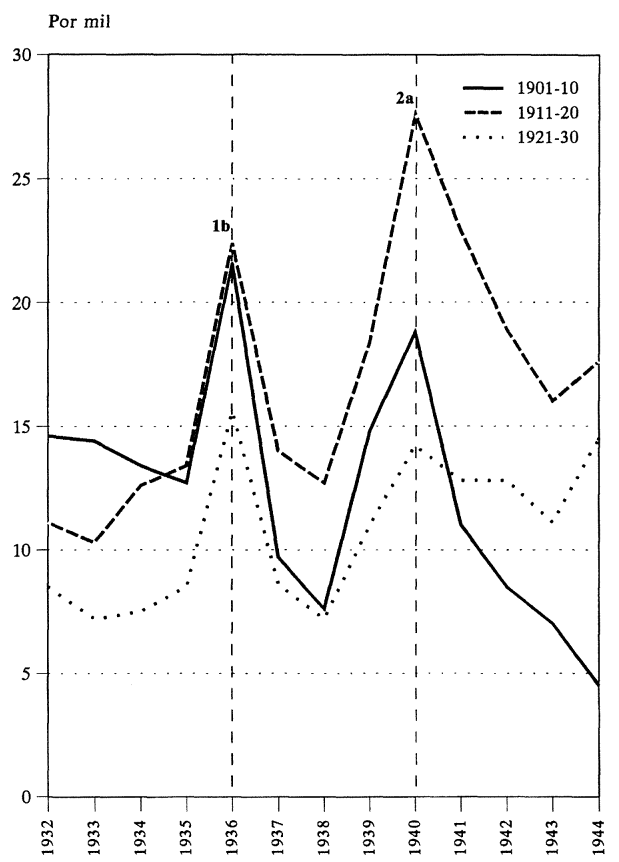

Fuente: Elaboración propia a partir de los datos primarios de la ESD.

FiguRA 1 bis.-Movilidad en el período de la guerra civil

Hubo dos picos de movilidad. El primero, en 1936, es debido tanto a la movilización militar (primera y segunda cohorte) como a los desplazamientos en busca de mayor seguridad, que afectan a las tres, aunque los nacidos en los años veinte (todavía muy jóvenes) se mueven junto con sus padres (1b). El segundo se produce en 1940, con la finalización del periodo bélico y una recuperación de la movilidad; se trata fundamentalmente de movilidad por motivos laborales o matrimoniales que había dejado de producirse durante el período de contienda civil, y se concentra a la finalización del mismo ${ }^{2}$. Esta recuperación está prota-

${ }^{2}$ No ha de olvidarse que al estar estudiando la primo-movilidad, no refleja la movilidad de «retorno», que significaría al menos un segundo movimiento, y que sería recogida al tratar movimientos de orden superior. 
gonizada fundamentalmente por los nacidos en la segunda década, que se encuentran en edad tanto de buscar empleo como de casarse en este momento, y que lo han retrasado durante el periodo bélico (2a).

Los años 1935 a 1950 representan una larga coyuntura desfavorable: desastre de la Guerra Civil, 2. ${ }^{a}$ Guerra Mundial y la rígida autarquía que se prolonga mucho más allá de la conflagración y esteriliza buena parte del esfuerzo productivo. Pero la década de los cincuenta ve el final de ese largo aislamiento y depresión económica. Comienza un período expansivo (1950-1974), que ha llegado a ser denominado como «los años del milagro económico» (Carreras, 1989). Pese a la idea generalizada de localización del gran éxodo migratorio en la década de los sesenta, éste empieza a despegar ya en la década anterior, como demuestran los saldos migratorios calculados por García Barbancho (1967) y Nadal (1984), en los que la migración neta para la provincia de Madrid, por ejemplo, es mayor en el segundo quinquenio de los años cincuenta que entre 1966 y 1970, y en la de Barcelona se aproxima considerablemente.

Los salarios industriales aumentaron, a partir de 1951, de forma mucho más rápida que los agrícolas, que sólo lo hicieron (y levemente) a partir de 1957 (Maluquer de Motes, 1989). Una nueva etapa de desarrollo urbano y de expansión industrial, de forma paralela a un mantenido proceso de expulsión de un excedente de mano de obra agrícola (jornaleros eventuales en su mayor parte, pequeños propietarios en menor medida) acelera el despoblamiento del campo e impulsa la concentración urbana. Entre 1950 y 1970 la población activa agraria masculina se reduce a la mitad (el campo pierde 1.300 .000 jornaleros) (Nicolau, 1989). La generación nacida en los años veinte, que se encuentra en torno a los 25 años al principio de la década, registra unas altas cotas de movilidad durante este período, en parte, como ya se ha señalado, por la concentración que produce el retraso de la misma hasta este momento (3a).

El final de los años cincuenta y la década siguiente registran unas corrientes migratorias no conocidas hasta el momento, ni por su intensidad ni por su extensión, como consecuencia del cambio de ciclo económico, en concreto, en torno al Plan de Estabilización de 1959 y sus efectos en los años siguientes. La generación nacida en la década de la Guerra Civil es la protagonista indudable de las migraciones interiores de la España de los sesenta; se la incluye, para comparar, en la figura 1 
(4a); presenta una alta concentración temporal del primer movimiento, y una alta intensidad en este período, debido al número de los individuos implicados. Pero lo que es más llamativo, es que incluso las generaciones más viejas muestran una nueva tendencia ascendente, una sobremovilidad, independiente de la edad en que se encuentran. La tercera cohorte, que había comenzado a reducir sus niveles de movilidad, ya entre los 30 y los 40 años cumplidos, no sólo frena este descenso sino que mantiene sus probabilidades de migración a niveles superiores a los que jamás llegó a alcanzar la primera de las cohortes $(3 b)$. La cohorte nacida durante la segunda década del siglo experimenta también una reactivación de las probabilidades de movilidad (2b).

Pero quizá el efecto más notable de la expansión industrial de los sesenta lo presenta la generación de la primera década del siglo. Nacidos 50 ó 60 años atrás muestran también una significativa reactivación de su movilidad, notable no sólo por la edad a la que se produce, sino por tratarse del primer movimiento (1c). Esta generación no sólo ha vivido las circunstancias más desfavorables del siglo, viendo afectadas sus probabilidades de movilidad, tanto en su intensidad como en su distribución, sino que además una parte de los individuos nacidos en este momento se vieron, cuando eran sus hijos y no ellos los que estaban en edad de migrar, haciendo las maletas y emigrando. Las causas que motivaron esta movilidad habrán de ser determinadas en posteriores etapas de esta investigación, pero sea una migración familiar en compañía de hijos en edad de buscar trabajo, o sea huyendo de una situación de penuria en busca de mejora, es sin lugar a dudas un comportamiento migratorio difícil de encontrar en otras cohortes menos marcadas por las circunstancias históricas.

Las décadas de los setenta y ochenta registran niveles muy bajos de migración para estas cohortes, tanto por causas estructurales como coyunturales. Los individuos estudiados son ya mayores y, por lo tanto, es de esperar que muestren bajas probabilidades de migración, alejados de las edades modales y de los motivos habituales. Pero la coyuntura económica de finales de los setenta y principios de los ochenta tampoco favoreció la movilidad, por lo que no se registraron para la segunda y tercera cohortes migraciones próximas a la edad de jubilación como ocurrió con la primera en los años sesenta; por otra parte, se debilitó el flujo emigratorio e incluso se inició un retorno desde Europa (no contabilizado por tratarse de segundo movimiento). Únicamente puede 
observarse una pequeña recuperación de la tendencia para los individuos de la primera cohorte en los años noventa $(1 d)$, pero éste es un fenómeno que puede deberse sobre todo a un efecto edad, lo que hace pensar que las generaciones más jóvenes presentarán un comportamiento similar hacia el 2000 y el 2010 (Abellán, 1993).

\section{Calendario migratorio}

Las tres cohortes presentan un calendario ${ }^{3}$ de movilidad similar, con la clásica curva de migración por edades (Rogers y Castro, 1981): una movilidad infantil relativamente alta acompañando a unos padres adultos-jóvenes migrantes, una clara edad modal en torno a los 25 años (aunque con altos niveles de movilidad entre los 20 y los 30) y un descenso de la misma menos brusco cuanto más joven es la cohorte (figura 2).

Este patrón migratorio responde a circunstancias ligadas a otros eventos biográficos, como son la búsqueda de empleo estable y el matrimonio, principales detonantes de movilidad, a pesar de las distintas coyunturas más o menos favorables vividas por los individuos de distintas edades de cada una de las cohortes.

Se aprecia una clara evolución en las diferentes cohortes hacia la concentración biográfica del primer movimiento (Figura 2,a). La generación más joven muestra un patrón más estandarizado, mientras la mayor (y en menor medida la segunda) presenta un patrón migratorio más laxo, apareciendo el primer movimiento más disperso a lo largo de toda su biografía. Estas generaciones de españoles evolucionaron, por la conjunción de causas biográficas e históricas, hacia una concentración de la primo-movilidad en torno a la edad modal (25-28 años), y una reducción de la dispersión cuanto más joven es la cohorte.

También se produce un proceso de ligero retraso de la misma. La movilidad de adolescentes se reduce progresivamente para los nacidos

${ }^{3}$ Se agrupan en cada edad todos los individuos de la cohorte que emigraron con esos años cumplidos; por ejemplo, un nacido en 1901 que emigra con cuatro años (en 1905), se contabiliza junto con otro nacido en 1904 que emigra también con cuatro años, en 1908. Por el contrario, en el apartado anterior, se contabilizaron en el mismo año, por ejemplo 1928, todos los movimientos que hicieron los individuos de una generación, por ejemplo la de 1901-10, que tenían en esa fecha entre 18 y 27 años cumplidos. 


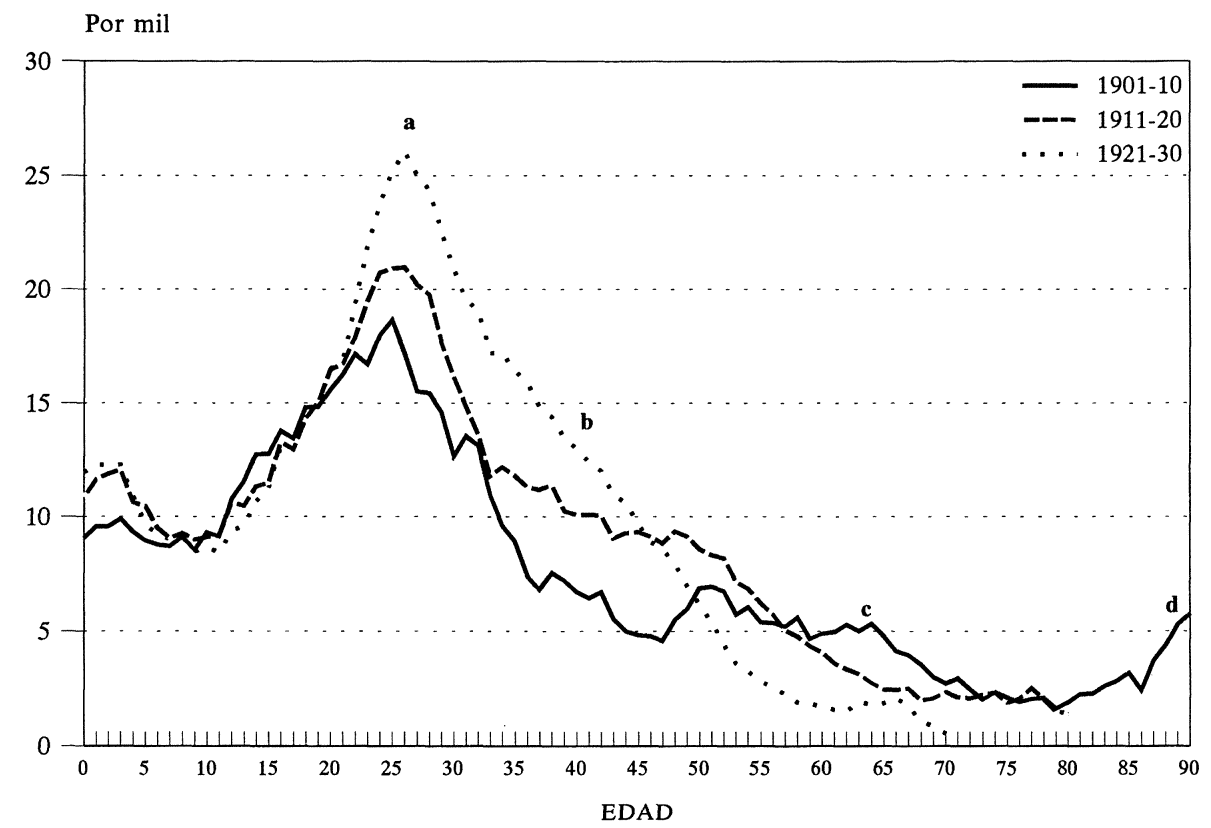

Fuente: Elaboración propia a partir de los datos primarios de la ESD.

FIgURA 2.-Probabilidades de primo-movilidad por edad

durante las tres primeras décadas del siglo, descendiendo las probabilidades de movilidad previa a los 20 años (10-20 años), mientras aumentan las probabilidades de primera migración adulta entre los 30 y los 45 años (b). En concreto, entre estas edades las dos cohortes más jóvenes mantienen probabilidades superiores al 10 por mil anual. Es decir, los españoles nacidos con posterioridad a 1910 no sólo retrasan su movilidad (reduciendo la movilidad precoz y concentrándola en las edades modales), sino que además la mantienen más alta hasta edades avanzadas.

Para la primera generación del siglo las probabilidades de realizar un primer movimiento migratorio descienden bruscamente a partir de los 30 años, manteniéndose bajas hasta alcanzar los 50 años. Para los nacidos entre 1911 y 1920, tras un descenso rápido, las probabilidades de movilidad adulta (posteriores a los 30 años) se estabilizan en niveles su- 
periores a los de la primera de las cohortes, manteniéndolos hasta los 50 años. La cohorte más joven mantiene de forma constante, a partir de los 25 años, niveles de movilidad superiores a los de las anteriores, hasta alcanzar los 45 años, edad a partir de la cual sus probabilidades de movilidad presentan niveles prácticamente nulos. Es decir, el aumento de la intensidad migratoria de las últimas cohortes no significó un aumento de la movilidad a edades jóvenes, sino que fue causado por un mantenimiento de las altas probabilidades migratorias entre los 30 y los 45 años; o lo que es lo mismo, fue ya de adultos cuando el conjunto de la cohorte superó sobradamente los niveles de movilidad de cohortes anteriores.

Este envejecimiento de la movilidad se ilustra en la figura 3, en la que se han representado las probabilidades de primo-movilidad de las tres

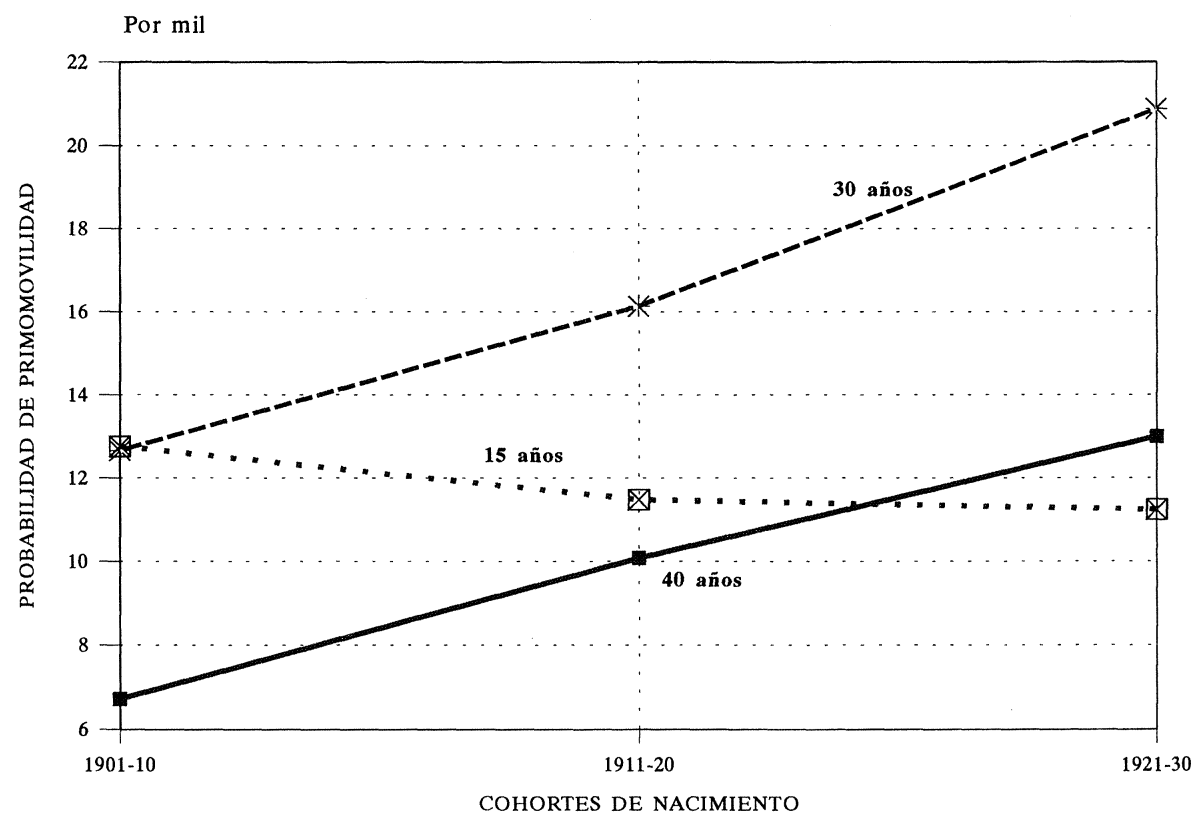

Fuente: Elaboración propia a partir de los datos primarios de la ESD.

FIGURA 3.-Probabilidades de primo-movilidad para algunas edades simples 
generaciones en tres momentos concretos de su curso de vida (a los 15, 30 y 40 años). Mientras la movilidad adolescente desciende suavemente, las probabilidades de movilidad adulta, tanto a los 30 como a los 40 años, presentan una continua tendencia ascendente. Como resultado de ello los cambios en el calendario de la migración son notorios. En la primera generación las probabilidades de migrar por primera vez a los 15 años se sitúan al mismo nivel que a los 30 , y las probabilidades de realizar un desplazamiento ya con 40 años son muy inferiores. En la cohorte de 1921-30, las pautas de movilidad en estos tres momentos biográficos han cambiado completamente: mientras a los 30 años muestran altas probabilidades migratorias en relación a las de la primera generación, a los 40 años sus probabilidades de primo-movilidad se sitúan al mismo nivel (13 por mil anual) que las probabilidades que tenían los sujetos de 15 y 30 años de la cohorte de 1901-10; y la probabilidad de primera migración con 15 años en el cohorte de 1921-30 presenta valores inferiores.

A partir de los 50 años las probabilidades de migrar se reducen considerablemente para la cohorte más joven, y a partir de los 55 para los nacidos en la segunda década (Figura 2). Por el contrario la generación más mayor, no sólo muestra una revitalización de la migración a partir de esta edad, sino que mantiene unos niveles relativamente destacables hasta los 65 años (c). Este fenómeno es, probablemente, producto de una conjunción de factores de momento histórico y edad. El final de la década de los cincuenta, y de la del sesenta propició un aumento de la movilidad en general, pero sobre todo de gente joven en busca de trabajo, es decir los hijos de los individuos nacidos en la primera década del siglo. Éstos, al mismo tiempo, se encontraban en edades próximas a la jubilación, lo que abre la posibilidad tanto de la migración familiar acompañando a los hijos, como de seguir su movimiento unos años después cuando éstos ya se han establecido, o de búsqueda de trabajo para ellos mismos. La atracción urbana e industrial de los años sesenta pudo llevar a algunos individuos a mover su hogar e iniciar el primer cambio residencial de su vida, con más de 50 años cumplidos.

Finalmente, en la primera de las cohortes (las dos siguientes son demasiado jóvenes en el momento de la encuesta para que se aprecie este fenómeno) destaca un claro efecto edad: una sobremovilidad significativa a partir de los 85 años $(d)$. Se trata, generalmente, de movi-

$$
-703-
$$


mientos de «reagrupación familiar» cuando el anciano ya no puede valerse por sí mismo y necesita de ayuda familiar ${ }^{4}$ (Abellán, 1993).

Edad media en la migración.-Para el cálculo de esta edad media (EMM) se han tomado tan sólo los primeros movimientos realizados por los individuos antes de los sesenta años, con la intención de poder comparar las cohortes estudiadas, dado que los nacidos en 1930 no sobrepasaban esa edad en el momento de la encuesta. Así los individuos de la primera generación del siglo se movieron, como media, a los 23.6 años, mientras que los nacidos en la década siguiente lo hicieron ya a los 24.4, presentando los más jóvenes un valor medio en torno a los 24 años.

La menor dispersión de la movilidad a lo largo del curso de vida, que presentan las generaciones más jóvenes, es el motivo de que la edad media no registre un mayor retraso en el calendario, como sería de esperar dada la evolución del perfil por edades, antes comentada, es decir, debido al ligero retraso del patrón migratorio que se produce entre la primera y la tercera de las cohortes. Éste no se ve reflejado en mayor medida en la EMM por el peso que en una media tienen siempre los valores extremos, en este caso, la movilidad a edades avanzadas que presentan los nacidos en la primera década (sobremovilidad entre los 50 y los 65 años), y altas probabilidades de migrar a edades infantiles (0-5 años) de las generaciones más jóvenes, lo que envejece el indicador para la primera cohorte, mientras lo rejuvenece para las dos siguientes.

Las probabilidades acumuladas de movilidad.-Éstas empiezan a distanciarse a partir de los 30 años (Figura 4). A dicha edad eran de 0.33, 0.36 y 0.38 respectivamente para las tres cohortes; es decir, mientras un $33 \%$ de los nacidos entre 1901 y 1910 se había movido con esa edad, de los nacidos 20 años más tarde se habían movido ya un $38 \%$. Pero estas mismas probabilidades de movilidad donde muestran una mayor diferencia entre las cohortes estudiadas es entre los 30 y los 50 años. Así, mientras a los 50 años un $42 \%$ de los individuos de la primera generación se había movido alguna vez, para la generación nacida dos décadas más tarde las probabilidades de movilidad se situaban 10 puntos por en-

4 Los movimientos de «institucionalización» o ingreso en residencias de ancianos no están recogidos, ya que la ESD se realizó tan sólo entre individuos residentes en viviendas familiares. 


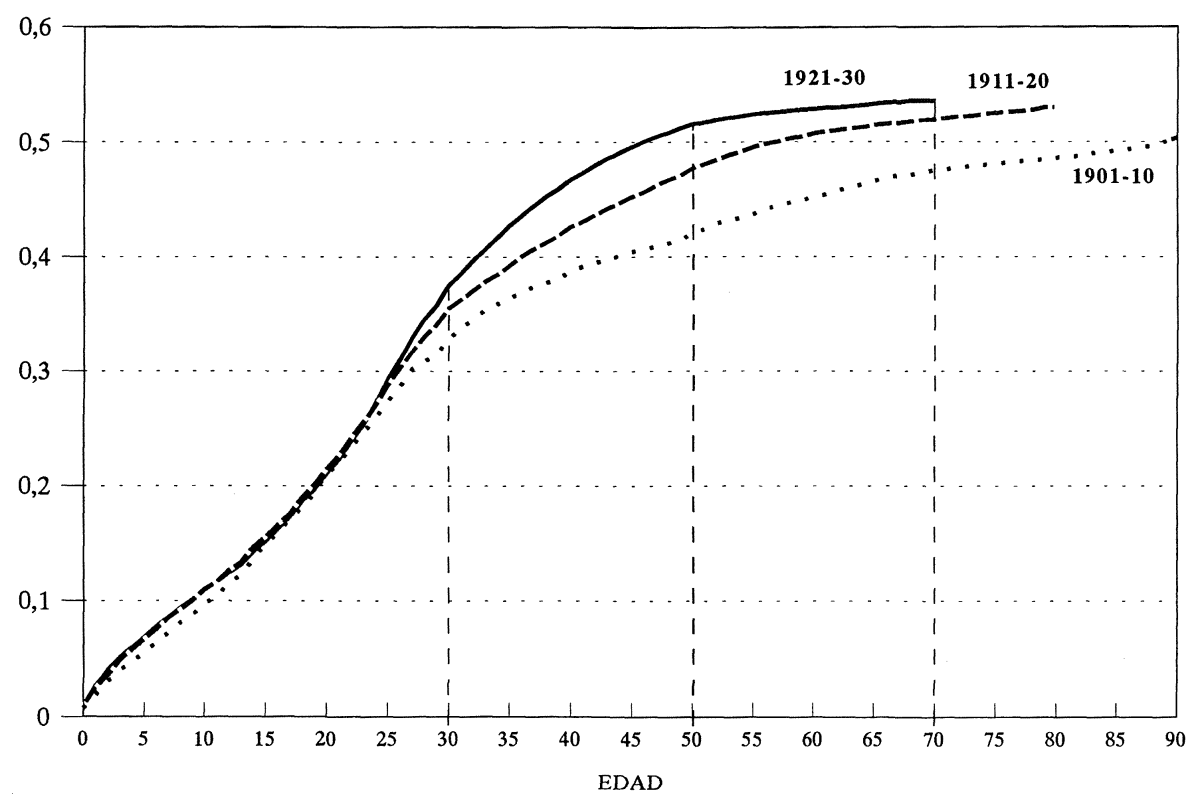

Fuente: Elaboración propia a partir de los datos primarios de la ESD.

FiguRa 4.-Probabilidades acumuladas de movilidad, según cohorte de nacimiento

cima. Lo que confirma el hecho, anteriormente apuntado, de que el verdadero aumento de la intensidad migratoria protagonizado por estas cohortes no significó tanto un mayor número de migrantes en las edades modales (en torno a 25 años), sino el mantenimiento de las altas cotas de migración de primer orden entre los 30 y los 45-50 años, edades en las que estas tres cohortes muestran comportamientos más diferenciales.

Tomando las probabilidades acumuladas tan sólo hasta los 70 años, con el fin de poder comparar las cohortes, se constata que sólo el $48 \%$ de los individuos nacidos en la primera década del siglo se habían movido alguna vez. Los individuos de la segunda generación habían alcanzado, a los 50 años, ese mismo nivel de movilidad de sus predecesores; mientras que la más móvil de las generaciones estudiadas, los nacidos en los 
años veinte, habían alcanzado ya un nivel de movilidad similar al de la primera cohorte 30 años antes, con 40 años cumplidos.

\section{Conclusiones}

Tan sólo la mitad, aproximadamente, de la población española nacida en las primeras décadas del siglo $\mathrm{xx}$ ha cambiado de municipio de residencia alguna vez en su vida; además, quien se ha movido lo ha hecho pocas veces. Este comportamiento sedentario se repite en las diferentes cohortes, lo que puede ser debido a una raíz cultural y económica (estructura productiva poco cambiante durante decenios), y por lo tanto de gran inercia.

Sin embargo, el número de individuos de cada cohorte que cambian de municipio de residencia, al menos una vez en la vida, es mayor cuanto más joven es la cohorte de nacimiento, es decir, muestran una intensidad migratoria creciente. Esta evolución hacia una mayor migrabilidad se vio favorecida por el devenir de los acontecimientos históricos. La coyuntura socioeconómica que conoció la generación más joven en torno a la edad de 30 años, posibilitó que sus probabilidades de migración se viesen mucho menos restringidas que las de generaciones anteriores.

Los españoles de la primera década del siglo presentan una menor concentración temporal de su movilidad, que fundamentalmente se produce a finales de la segunda década y en los primeros años veinte, en parte como movilidad de hogar, acompañando a sus padres migrantes y como entrada temprana en el mercado de trabajo. Fue una movilidad favorecida por el desarrollo industrial del momento y de la pequeña «revolución económica» producida por la guerra europea con el consiguiente aumento de las exportaciones; pero la coyuntura favorable no duró mucho, y hacia finales de esta misma década sus movimientos migratorios se ven frenados. Los individuos nacidos entre 1911 y 1920 no vivieron las mismas circunstancias, marcados por la Guerra Civil y forzados a retrasar su primera migración hasta el final de la misma, y concentrarla en los difíciles años cuarenta, lo que se traduce en una mayor movilidad adulta. La generación nacida en los años veinte mantiene altas probabilidades de realizar un primer movimiento migratorio durante las décadas económicamente expansivas (años cincuenta y se- 
senta), al coincidir expansión económica y edad habitual de migración. Las favorables condiciones económicas conocidas por las cohortes más jóvenes probablemente son la causa de los cambios en el calendario de la movilidad, que favoreció el mantenimiento de altas probabilidades de primo-movilidad hasta edades más avanzadas.

El patrón migratorio de las tres cohortes registra una concentración creciente de la movilidad en torno a la edad modal (25 años), migración ligada fundamentalmente con el mercado laboral y matrimonial; las generaciones más jóvenes presentan un patrón mucho más estandarizado para los primeros movimientos, frente a la dispersión biográfica de los mismos que registran las otras. También se puede observar un desplazamiento de la movilidad hacia edades más tardías (30-45 años); es decir, un ligero envejecimiento del patrón migratorio. Ello puede ser debido a una influencia tanto de factores biográficos como históricos: evolución hacia una edad de entrada en el mercado laboral más tardía; los condicionamientos históricos potenciaron una movilidad temprana para la primera generación, mientras para la última motivaron un retraso de la misma.

$\mathrm{El}$ aumento de la intensidad migratoria no lo protagonizaron, por tanto, los jóvenes, sino que fue siendo ya adultos, con edades comprendidas entre los 30 y los 45 años, cuando los individuos pertenecientes a las cohortes más tardías, manteniendo altas probabilidades de primo-movilidad, convirtieron a éstas en generaciones «más migratorias».

Finalmente, existe un comportamiento migratorio diferencial en la primera de las cohortes de nacimiento: una recuperación de las probabilidades de movilidad entre los 50 y los 65 años, que no se encuentra para ninguna de las cohortes posteriores. Muchos individuos de esa generación han realizado su primer movimiento migratorio, en busca de trabajo asalariado o como migración familiar con hijos en edad de trabajar, con más de 50 años cumplidos.

\section{BIBLIOGRAFÍA}

ABellán García, A. (1993): «La decisión de emigrar en las personas de edad». Estudios Geográficos, n. 210, p. 5-17.

Blanes, A.; Gil, F.; PÉrez, J. (1996): Población y actividad en España: evolución y perspectivas. Barcelona, La Caixa, 242 p. 
Bocquet-Appel, J. P.; Courgeau, D.; Pumain, D. (1996): Spatial Analysis of Biodemographic Data. London, J. Libbey Eurotext, 367 p.

CABO ALONSO, A. (1961): «Valor de la inmigración madrileña». Estudios Geográficos, n. 8485, p.353-374.

CAPEL SÁEZ, H. (1967): «Los estudios acerca de las migraciones interiores en España». Revista de Geografia, n. 1, p. 77-101.

CARRERAS, A. (coord.)(1989): Estadísticas históricas de España, siglos XIX-XX. Madrid, Fundación Banco Exterior, 632 p.

CARRERAS, A. (1989): «Industria», en Carreras: Estadísticas históricas...; p. 169-248.

Courgeau, D. (1988): Méthodes de mesure de la mobilité spatiale. Paris, INED, $301 \mathrm{p}$.

Courgeau, D.; LELIĖvre, E. (1989): Analyse démographique des biographies. Paris, INED, $268 \mathrm{p}$.

GARCíA BARBANCHO, A. (1967): Las migraciones interiores en España. Estudio cuantitativo desde 1900. Madrid: Instituto de Desarrollo Económico, 128 p. y tablas.

GARCÍA FERNÁNDEZ, J. (1956): «La atracción demográfica de Madrid». Estudios Geográficos, n. 62 , p. $87-91$

GARCÍA FERNÁNDEZ, J. (1965): La emigración exterior de España. Barcelona, Ariel, 302 p.

GARRIDO, L.J. (1992): Las dos biografías de la mujer en España. Madrid, Instituto de la Mujer, $310 \mathrm{p}$.

GonZÁLEZ-RothVos y GiL, M. (1954): «La emigración española a Iberoamérica». Estudios Demográficos, vol. 3, p.315-407.

LELIÈVRE, E. (1990): «Bilan des connaissances sur la mobilité individuelle au cours du cycle de vie»; p. 49-63. En C. Bonvalet et A. M. Fribourg: Strategies résidentielles. Paris, INED, $459 \mathrm{p}$.

MALUQUER De Motes, J. (1989): «Precios, salarios y beneficios. La distribución funcional de la renta», en Carreras: Estadísticas históricas...; p. 495-532

NADAL, J. (1984): La población española (siglos XVI a XX). Barcelona, Ariel, $267 \mathrm{p}$.

NicolaU, R. (1989): «Población», en Carreras: Estadísticas históricas...; p. 49-90.

Pujadas, I.; García Coll, A.; Puga González, M. D. (1995): «Migraciones interiores en España: tendencias recientes y perspectivas de futuro (1970-2001)». Revista de Geografía, 29, n. 3, p. 9-150.

PuYol ANTOLÍn, R. (1979): Emigración y desigualdades regionales en España. Madrid, EMESA, $238 \mathrm{p}$.

RÓDENAS CALATAYUd, C. (1994): Emigración y Economía en España. Madrid, Civitas, 299 p.

Rodríguez GaLDo, M. X. (1995): O fluxo migratorio dos séculos XVIII ó XX. Santiago de Compostela, Xunta de Galicia, $126 \mathrm{p}$.

Rogers, A.; CASTRO, L. J. (1981): Model Migration Schedules in Multistate Demographic Analysis: the Case of Migration. Laxemburg (Austria), International Institute for Applied Systems Analysis, $120 \mathrm{p}$.

SÁNCHEZ JiMÉnEZ, J. (1984): «La población, el campo y las ciudades». En (R. Menéndez Pidal) Historia de España, t. XXXVII, p. 173-433.

TAPINOS, G. (1966): «Migrations et particularismes regionaux en Espagne». Population XXI, n. 6 ; p. 1135-1164

REsumen: Análisis del fenómeno migratorio desde la óptica del curso de vida de sus protagonistas: es un estudio longitudinal de la historia migratoria de las tres primeras cohortes del siglo (los nacidos en 1901-10, 1911-20 y 1921-30), y más en concreto de su primer movimiento, que para la mayoría de ellos fue el único y para casi todos el más importante y de mayores consecuencias biográficas. Se trata de situar la movilidad en el momento cronológico o histórico en que se produce, y en el momento biográfico o edad del sujeto en el cambio de municipio de residencia. 
PALABRAS ClAVE: Movimientos migratorios, estudio longitudinal, curso de vida, evento biográfico, calendario migratorio, primo-movilidad, movilidad residencial, Encuesta Sociodemográfica 1991, España.

ABSTRACT: This paper approaches the analysis of the migratory process from the life course perspective; it presents a longitudinal study of the migration history of the three cohorts born at the beginning of the century (1901-10, 1911-20, 1921-30), focusing on the first migratory move, which for most of the individuals was the only one and the most influential in their life course trajectory. It aims at placing this mobility both within the historical context in which it took place and within the biographical stage of the migrant.

KEY WORDS: Migration, longitudinal study, life course, life event, migratory calendar, first move, residential mobility, Sociodemographical Survey 1991, Spain.

RESUMÈ: Analyse du procès migratoire dès la perspective de la course de vie; c'est une étude longitudinal de l'histoire migratoire des trois premières cohortes du siècle (190110, 1911-20, 1921-30), plus précisément de leur premier mouvement, qui fut l'unique pour la majorité et le plus important avec des grandes conséquences biographiques. Il s'agit de situer cette mobilité dans le moment historique où elle se développe et dans le moment biographique de l'individu.

MOTS CLÉ: Mouvements migratoires, étude longitudinal, course de vie, événement biographique, calendrier migratoire, premier mouvement, mobilité residentielle, Enquête Sociodémographique 1991, Espagne. 